\title{
LIGHTLY COMPACT SPACES AND INFRA $H$-CLOSED SPACES
}

\author{
DARRELL W. HAJEK AND AARON R. TODD
}

\begin{abstract}
We show that a topological space is lightly compact if and only if each countable open filter base has a cluster point. This gives a direct connection with $H$-closed spaces, and suggests the definition of infra $H$-closed spaces as those topological spaces whose continuous images in first countable Hausdorff spaces are closed. These properties are distinct and provide another characterization of pseudocompact $T_{3 \frac{1}{2}}$ spaces.
\end{abstract}

In [1] light compactness was used to characterize pseudocompact completely regular spaces, and in [7] it was used in obtaining several filter characterizations of quasi-regular Baire spaces. In this paper we obtain a filter characterization of light compactness and several consequences including a closedness property related to $\mathrm{H}$-closure.

A space $X$ is called $H$-closed if for any Hausdorff space $Y$ and any continuous $f: X \rightarrow Y, f[X]$ is closed in $Y$. A space $X$ is called lightly compact if every locally finite collection of open subsets of $X$ is finite. It is well known (see [6]) that $H$-closed spaces can be characterized as those for which every open cover contains a finite subcollection whose union is dense. There is a striking similarity between this and the following characterization of light compactness which appears in [3]. A space is lightly compact if and only if every countable open cover contains a finite subcollection whose union is dense.

We now obtain another characterization of light compactness. By an open filter base in a space we will mean a nonempty collection of nonempty open sets such that the intersection of any two elements of the collection contains an element of the collection. If $\mathcal{F}$ is an open filter base in a space $X$, we will say that a point $x \in X$ is a cluster point of $\mathcal{F}$ if $x \in \bigcap\left\{\mathrm{cl}_{X}(U): U \in \mathcal{F}\right\}$.

Received by the editors March 10, 1973 and, in revised form, February 10, 1974.

AMS (MOS) subject classifications (1970). Primary 54D30; Secondary 54D20, 54D25.

Key words and phrases. Lightly compact spaces, open filter base, $H$-closed, absolutely closed, pseudocompact. 
Theorem 1. A space $X$ is lightly compact if and only if each countable open filter base in $X$ has a cluster point.

Proof. Let $\left\{U_{n}: n=1,2, \cdots\right\}$ be a countable open filter base in a space $X$, and suppose that $\bigcap_{n=1}^{\infty} \operatorname{cl}_{X}\left(U_{n}\right)=\varnothing$. Then $\left\{X \sim \operatorname{cl}_{X}\left(U_{n}\right)\right.$ : $n=1,2, \cdots\}$ is a countable open cover of $X$, but the complement of each $X \sim \mathrm{cl}_{X}\left(U_{k}\right)$ contains an element of $\left\{U_{n}: n=1,2, \cdots\right\}$. Hence the union of any finite subcollection of $\left\{X \sim \operatorname{cl}_{X}\left(U_{n}\right): n=1,2, \ldots\right\}$ cannot be dense in $X$; so $X$ is not lightly compact. Suppose now that $\left\{V_{n}: n=1,2, \ldots\right\}$ is a countable open cover of $X$ the union of no finite subset of which is dense. Now $\left\{X \sim \bigcup_{k=1}^{n} \operatorname{cl}_{X}\left(V_{k}\right): n=1,2, \ldots\right\}$ is a countable open filter base in $X$. Given any $x \in X$, there is some $n$ such that $x \in V_{n}$. Then $x \notin$ $\mathrm{cl}_{X}\left(X \sim \mathrm{cl}_{X}\left(V_{n}\right)\right)$; so

$$
x \notin \bigcap_{m=1}^{\infty}\left(\operatorname{cl}_{X}\left(X \sim \bigcup_{k=1}^{m} \mathrm{cl}_{X}\left(V_{k}\right)\right)\right) .
$$

Thus $\left\{X \sim \bigcup_{k=1}^{n} \mathrm{cl}_{X}\left(V_{n}\right): n=1,2, \cdots\right\}$ has no cluster point.

Theorem 1 allows us to establish quite easily a result about continuous images of lightly compact spaces.

Proposition 2. Suppose $X$ is lightly compact and $f: X \rightarrow Y$ is continuous. Then any subspace $Z$ of $Y$ such that $f[X] \subseteq Z \subseteq \operatorname{cl}_{Y}(f[X])$ is lightly compact.

Proof. Suppose $\left\{V_{n}: n=1,2, \cdots\right\}$ is an open filter base in $Z$. Then $\left\{f^{-1}\left[V_{n}\right]: n=1,2, \cdots\right\}$ is a countable open filter base in $X$, which, by Theorem 1 , must have a cluster point $x$. If $f(x)$ were not a cluster point of $\left\{V_{n}: n=1,2, \ldots\right\}$ then there would exist an open neighborhood $U$ of $x$ disjoint from some $V_{n}$, and this would imply that $f^{-1}[U]$ is an open neighborhood of $x$ disjoint from $f^{-1}\left[V_{n}\right]$. Thus $f(x)$ must be a cluster point of $\left\{V_{n}: n=1,2, \cdots\right\}$ and $Z$ must be lightly compact by Theorem 1 .

Now Proposition 2 gives us another characterization of lightly compact Hausdorff spaces.

Theorem 3. A Hausdorff space $X$ is lightly compact if and only if for each Hausdorff space $Y$ and each continuous $f: X \rightarrow Y$, no point $y \in$ $\mathrm{cl}_{Y}(f[X]) \sim f[X]$ has a countable neighborhood base.

Proof. Let $f$ be a continuous function from any space $X$ into a Hausdorff space $Y$ and suppose that $x \in \mathrm{cl}_{Y}(f[X]) \sim f[X]$ has a countable neighborhood base $\left\{U_{n}: n=1,2, \cdots\right\}$. Then $\left\{f[X] \cap U_{n}: n=1,2, \cdots\right\}$ is a countable open filter base in $f[X]$ which has no cluster point. Thus $f[X]$ 
and, hence, $X$ are not lightly compact. Let us now suppose that $X$ is a Hausdorff space which is not lightly compact. By Proposition 2 there is a countable open filter base $\left\{U_{n}: n=1,2, \ldots\right\}$ in $X$ which has no cluster point. Let $X^{*}$ denote the set $X \cup\{w\}$ (where $w$ is an object not already in $X)$. With the topology generated by the open subsets of $X$ together with the sets of the form $U_{n} \cup\{w\}, X^{*}$ is clearly a Hausdorff space. It is also clear that the embedding of $X$ in $X^{*}$ is continuous, that $w$ is not in the image of $X$ but is in its closure, and that $w$ has a countable neighborhood base.

In view of the remarkable similarity in the characterizations of $H$-closed spaces and lightly compact spaces one is tempted to try to characterize lightly compact spaces in terms of the spaces in which their continuous images are closed. Theorem 3 would seem to indicate the natural direction to try.

Definition. A space $X$ will be called infra $H$-closed provided that any continuous image of $X$ in any first countable Hausdorff space is closed.

Proposition 2 and Theorem 3 clearly show that lightly compact implies infra $H$-closed, and this fact together with the construction of $X^{*}$ in the proof of Theorem 3 (in particular the obvious fact that $X$ first countable implies $X^{*}$ first countable) give us the following result.

Proposition 4. If $X$ is a first countable Hausdorff space then $X$ is lightly compact if and only if $X$ is infra $\mathrm{H}$-closed.

We may also enlarge on the characterization of completely regular pseudocompact spaces given in [1].

Theorem 5. If $X$ is a completely regular space the following are equivalent.

(i) $X$ is pseudocompact,

(ii) $X$ is lightly compact,

(iii) $X$ is infra $H$-closed.

Proof. The equivalence of (i) and (ii) is Theorem 3 of [1], and we have just noted that (ii) implies (iii). If $X$ is not pseudocompact it is easily seen that there is a continuous real valued function on $X$ whose image is not closed, and, hence, that $X$ is not infra $H$-closed.

It is not, however, the case that lightly compact and infra $\mathrm{H}$-closed are equivalent in general. In [4] Herrlich, beginning with a $T_{1}$ space $Y$, constructs a regular Hausdorff space $X$ such that the only continuous functions from $X$ into $Y$ are constant. His construction involves the cardinality of $Y$, but only as the cardinality of collections of open sets whose intersections are singletons. Thus only the most trivial modifications are needed in his 
argument to produce a regular Hausdorff infra $H$-closed space. It is also easily shown that the space constructed in this manner is not lightly compact.

In [5] an $H$-closed (in fact minimal Hausdorff) space is constructed which is of first category in itself. As regular $H$-closed spaces are compact, this example is not regular. Any regular lightly compact space must be a Baire space [7, Lemma 3], and thus it is of interest to note that the space constructed in [4] can be shown to be the countable union of nowhere dense subsets using essentially the same argument as can be used to show it is not lightly compact.

For those interested, we would like to note that ideas related to those in this paper are surveyed in [2].

\section{REFERENCES}

1. R. W. Bagley, E. H. Connell and J. D. MacKnight, Jr., On properties characterizing pseudo-compact spaces, Proc. Amer. Math. Soc. 9 (1958), 500-506. MR $20 \# 3523$.

2. M. P. Berri, J. R. Porter and R. M. Stephenson, Jr., A survey of minimal topological spaces, General Topology and its Relations to Modern Analysis and Algebra III (Proc. Conf. Kanpur, 1968), Academia, Prague, 1971, pp. 93-114. MR $43 \# 3985$.

3. J. Colmez, Sur les espaces précompacts, C. R. Acad. Sci. Paris 233 (1951), 1552-1553. MR 14, 1106.

4. H. Herrlich, Wann sind alle stetigen Abbildungen in $Y$ konstant?, Math. $Z$. 90 (1965), 152-154. MR 32 \#3029.

5. - Nicht alle $T_{2}$-minimalen Räume sind von 2 Kategorie, Math. Z. 91 (1966), 185. MR 32 \#6392.

6. C. Liu, Absolutely closed spaces, Trans. Amer. Math. Soc. 130 (1968), 86-104. MR $36 \# 2107$.

7. R. A. McCoy, A filter characterization of regular Baire spaces, Proc. Amer. Math. Soc. 40 (1973), 268-270.

DEPARTMENT OF MATHEMATICS, UNIVERSITY OF PUERTO RICO, MAYAGÜEZ, PUERTO RICO 00708 (Current address of D. W. Hajek)

Current address (A. R. Todd): Department of Mathematics, Brooklyn College (CUNY), Brooklyn, New York 11210 